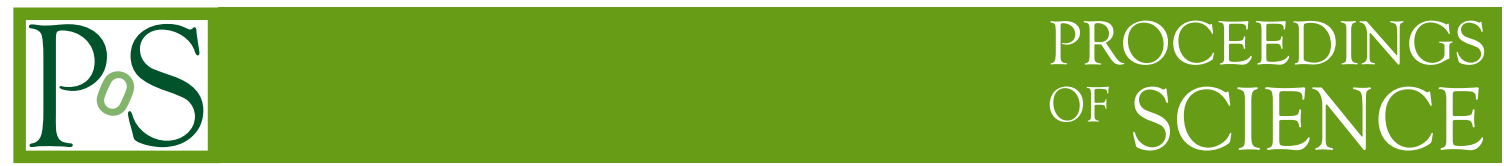

\title{
Search for new physics in events with two photons, many jets, and low missing transverse energy
}

\section{Marc Weinberg*}

Florida State University

E-mail: marc.gabriel.weinbergecern.ch

\begin{abstract}
Many supersymmetric and exotic models of new physics predict high-multiplicity events with leptons or electroweak gauge bosons and low missing transverse energy in the final state. We present results of a search for new physics at CMS in events with two photons and many hadronic jets with no requirement for large missing transverse energy.
\end{abstract}

36th International Conference on High Energy Physics,

July 4-11, 2012

Melbourne, Australia

\footnotetext{
* Speaker.
} 


\section{Introduction}

Many SUSY searches rely on signatures with large $\mathbb{E}_{T}$ from the presence of a heavy, stable lightest SUSY particle (LSP). Consequently, many low- $\mathbb{Z}_{T}$ topologies remain relatively unexplored at the LHC, and these become more interesting as the available phase space for high- $\mathbb{E}_{T}$ SUSY models is reduced by recent LHC results [1]. Several well motivated SUSY models, often characterized by $R$-parity violation, gauge mediated SUSY breaking, or light hidden sectors, predict low $E_{T}$ signatures.

Without $\mathbb{E}_{T}$ as a handle on the event, new analysis techniques are required in order to reduce the SM background. By analogy with searches for high multiplicity final states from black holes, we define $S_{\mathrm{T}}$ as the scalar sum of the transverse momenta $p_{\mathrm{T}}$ of all photons and jets in the event, as well as any $E_{T}$ (for events with $\not_{T}>20 \mathrm{GeV}$ ). Due to their generality, searches based on high- $S_{\mathrm{T}}$ signatures are sensitive to a wide range of new physics models.

\section{Stealth supersymmetry}

Stealth SUSY [2] provides a natural mechanism for producing low- $\mathbb{H}_{T}$ SUSY topologies without the need for fine tuning or $R$-parity violation. An additional hidden sector is introduced at the weak scale, populated, in the simplest case, by a singlet state $S$ and its "singlino" superpartner $\widetilde{S}$. If SUSY is broken at a scale below this hidden sector, as is natural in models with gauge-mediated SUSY with a gravitino $(\widetilde{G})$ LSP, then the hidden sector spectrum can be nearly supersymmetric. As a result, $S$ and $\widetilde{S}$ can be very nearly mass degenerate, and no special tunings of masses are required. The gravitino LSP, which emerges from the decay $\widetilde{S} \rightarrow \widetilde{G} S$, can be both light and soft, resulting in a final state with low $\mathbb{E}_{T}$. The full cascade decay is shown in Fig. 1.

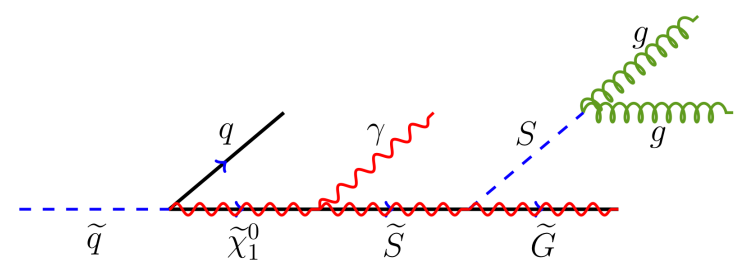

Figure 1: Decay of a squark in stealth SUSY.

We consider a class of stealth SUSY models based on the T2 simplified SUSY model [3]. To this model we add an additional hidden sector comprising a singlet state $S$ and a singlino $\widetilde{S}$. The gluino mass $m_{\widetilde{g}}$ is set to $1.5 \mathrm{TeV}$. In addition, we apply a number of simplifying assumptions: The singlet / singlino masses $m_{S} / m_{\widetilde{S}}$ are set to $90 \mathrm{GeV}$ and $100 \mathrm{GeV}$, respectively. The mass of the neutralino $m_{\widetilde{\chi}_{1}^{0}}$ is constrained to half the mass of the squark, $m_{\widetilde{q}}$, and its branching ratio to a photon via $\widetilde{\chi}_{1}^{0} \rightarrow \gamma \widetilde{S}$ is required to be 1 .

The cross sections for each of these model points, as well as the uncertainties on those cross sections (from scale, PDF, and $\alpha_{s}$ uncertainty) are determined at next-to-leading order + nextto-leading logarithmic (NLO + NLL) accuracy [4]. The acceptances for each of the dominant 
subprocesses is determined at lowest order (LO) from PYTHIA, and an NLO / LO $K$-factor is applied by subprocess. The selected final states are required to have 2 reconstructed photons and at least 2 reconstructed jets.

\section{Analysis overview}

The signal processes are expected to contain a large number of jets, and this analysis relies on our ability to predict the number of background events in high jet multiplicity final states based on a low multiplicity sideband. For this purpose we employ the variable $S_{\mathrm{T}}$, defined as the scalar sum of the transverse momenta of all photons and jets in the event, as well as any missing transverse energy:

$$
S_{\mathrm{T}}=\not B_{T}+\sum_{\gamma} p_{\mathrm{T}}+\sum_{\text {jets }} p_{\mathrm{T}}
$$

The shape of this variable has been shown to be independent of object multiplicity for QCD in a previous CMS search for black holes [5], a phenomenon referred to here as " $S_{\mathrm{T}}$ scaling", as shown in Fig. 2.

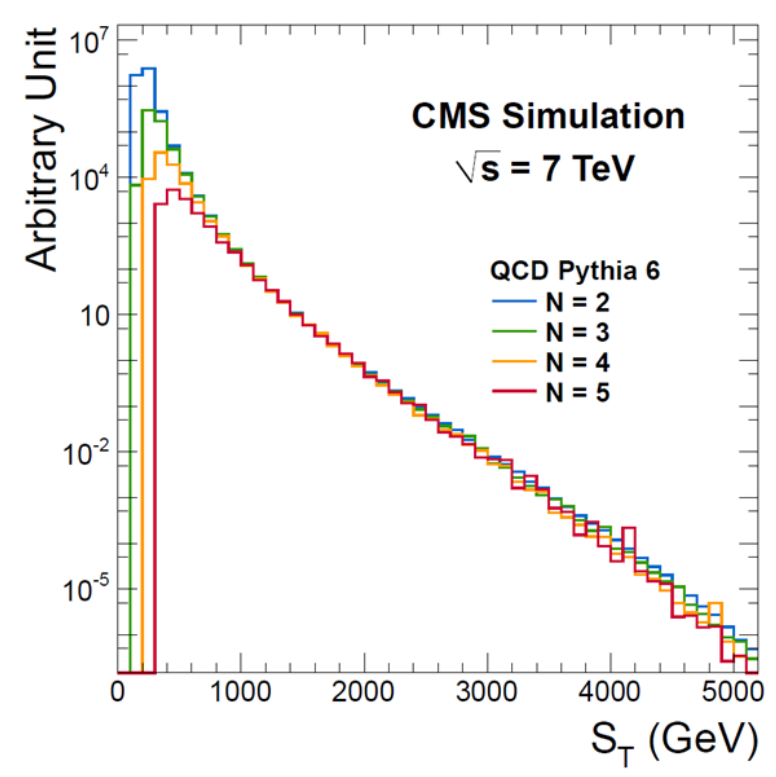

Figure 2: $S_{\mathrm{T}}$ distributions obtained from Pythia6 QCD Monte Carlo for four exclusive multiplicities of 2, 3, 4 , and 5. Shapes are independent of the multiplicity of the final state.

The black hole analysis is concerned with general object multiplicity, so the dominant contribution to the background comes from QCD. This analysis additionally requires the presence of two photons, so it must be verified that $S_{\mathrm{T}}$ scaling continues to hold for final states including photons. For this we consider a large control sample from data with 1 photon + jets. Fig. 3 shows the $S_{\mathrm{T}}$ distribution for this sample, from which we conclude that the $S_{\mathrm{T}}$ shape is indeed consistent, with no dependence on jet multiplicity. In addition, this exercise was performed on a MC sample of diphoton events produced with MADGRAPH, for which similar results were obtained. 

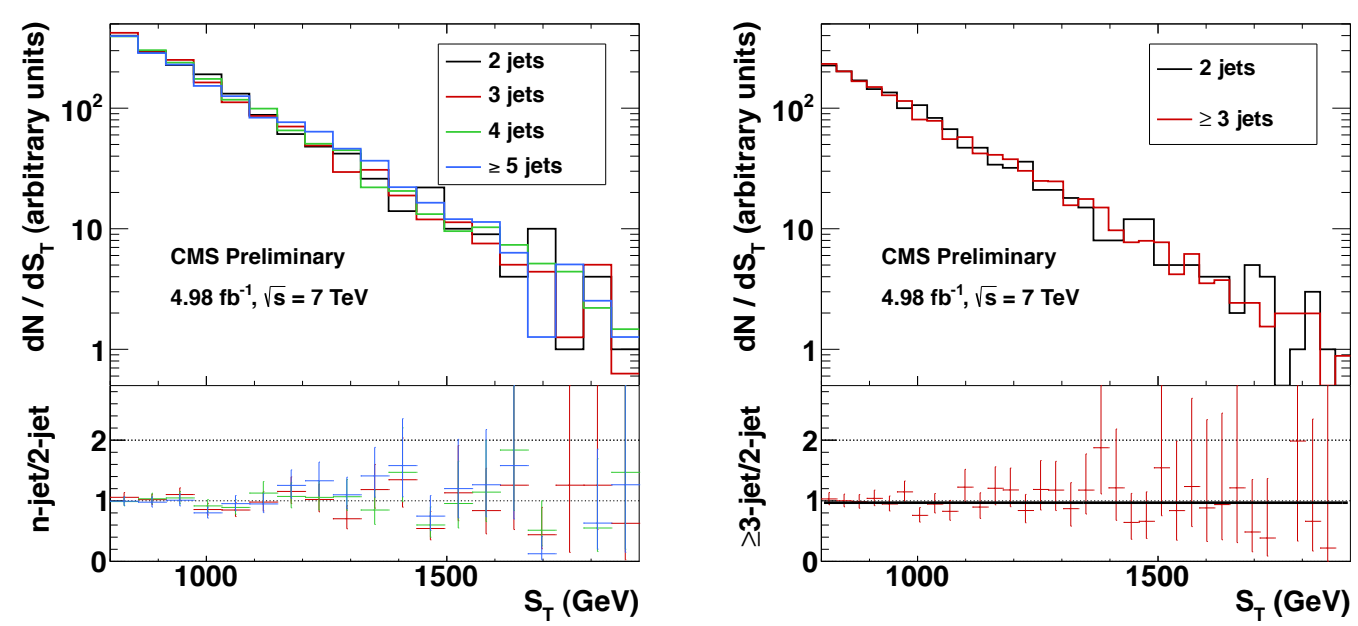

Figure 3: $S_{\mathrm{T}}$ spectra from the photon + jets data control sample, area-normalized for $S_{\mathrm{T}}>800 \mathrm{GeV}$. We show (a) the spectra for events with two, three, four, and five or more jets along with the $n$-jet / 2 -jet ratio where $n=3,4$, or $\geq 5$; and (b) the spectra for events with two and three or more jets along with the $\geq 3$-jet / 2-jet ratio.

Given $S_{\mathrm{T}}$ scaling, it is possible to determine the shape of the distribution in a "jet-multiplicity sideband", comprising the 2- and 3-jet bins in this analysis. This shape can then be applied to the signal region, taken here to be the 4- and $\geq 5$-jet bins. The signal is also expected to have large values of $S_{\mathrm{T}}$, so the overall normalization can then be determined for this region via an $S_{\mathrm{T}}$ sideband, taken to be $600 \mathrm{GeV}<S_{\mathrm{T}}<700 \mathrm{GeV}$.

From this normalized background shape, we then determine the expected number of background events with an $S_{\mathrm{T}}$ above some threshold optimized for each squark mass. Finally, we perform a simple counting experiment to test for an excess over the expected background.

\section{Background estimate}

The shape of the $S_{\mathrm{T}}$ curve is estimated from the background-dominated 2- and 3-jet bins. The events in these bins are fit to the nominal functional form $1 / x^{p}$, where $x \equiv S_{\mathrm{T}} / \sqrt{s}$. In addition, these events are fit to two other functions, $e^{p x}$ and $1 / x^{p_{0}+p_{1} \log x}$. The difference between these fitted curves and the nominal curve is used to estimate the uncertainty due to choice of fitting function. The results of all three functional fits are shown in Fig. 4. All three functions give consistent results, and the maximum difference between them is taken to be the systematic uncertainty.

This shape is then normalized in the $S_{\mathrm{T}}$ sideband $600 \mathrm{GeV}<S_{\mathrm{T}}<700 \mathrm{GeV}$. The total event count is determined from data for all events with $\geq 4$ jets, and the fraction of those events in the 4-jet bin is determined from the ratio of the two event counts. Results for this ratio are consistent between data and MC. The normalized $S_{\mathrm{T}}$ shape for the two jet bins, 4-jet and $\geq 5$-jet, is shown in Fig. 5, along with the normalization region used and the distribution for a representative signal MC point. 


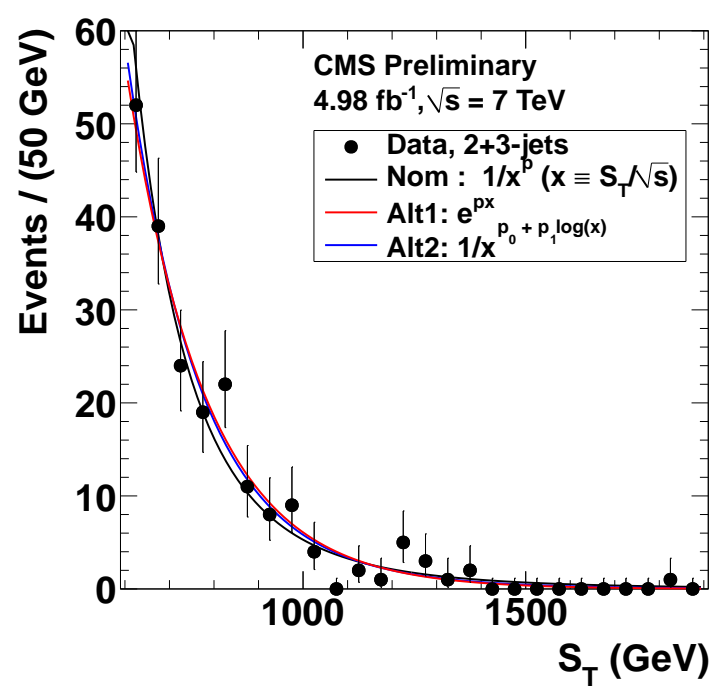

Figure 4: Fit of three functional forms to the $S_{\mathrm{T}}$ distribution in events with two or three jets. The nominal function is $1 / x^{p}$; the two other functions are used to estimate the systematic uncertainty related to the choice of fit function.
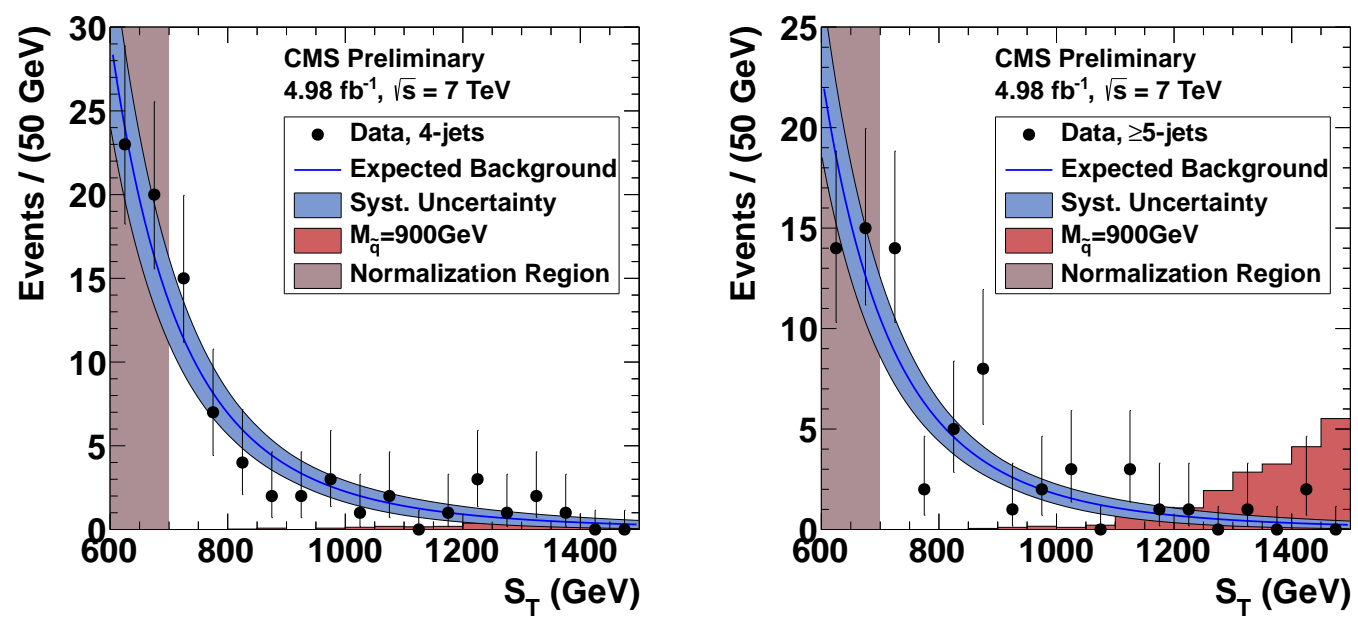

Figure 5: Data and background expectation with uncertainty in events with four jets (left) and $\geq 5$ jets (right). 


\section{Results and conclusions}

The $S_{\mathrm{T}}$ distribution for all data with 2 photons and at least 4 jets is shown in Fig. 6. Good agreement is observed between the data and the background projection from the fit procedure. There is no evidence of an excess of events over the background, and we calculate a limit on the signal cross section via a counting experiment. The total number of events above a given $S_{\mathrm{T}}$ threshold is counted, with the threshold determined individually for each signal mass point to optimize the limit.

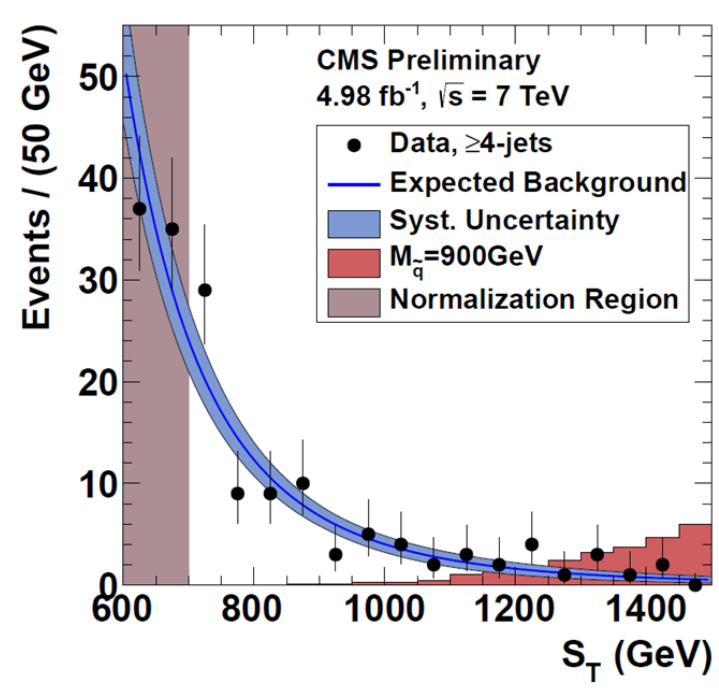

Figure 6: Data and background expectation with uncertainty in events with $\geq$ four jets.

A 95\% CL limit was set on the squark mass in the stealth SUSY class of models using the $\mathrm{CL}_{\mathrm{s}}$ method. The results are shown in Fig. 7. Here, the dotted line indicates the median expected cross section limit, and the green band shows the $\pm 1 \sigma$ experimental uncertainty on the limit. The dark red line gives the predicted theory cross section of the signal, and the red band around it shows the $\pm 1 \sigma$ theory uncertainty. Thus, we exclude all squark masses in this class of models out to the intersection of the cross section limits and the conservative edge of the predicted cross section. This yields an expected limit of $M_{\tilde{q}}>1420 \mathrm{GeV}$ and an observed limit of $M_{\tilde{q}}>1430 \mathrm{GeV}$.

This analysis presents a new search methodology based on low- $\mathbb{E}_{T}$ topologies. We investigate events with 2 photons, 4 or more jets, and $S_{\mathrm{T}}>700 \mathrm{GeV}$, with no requirement on $\mathbb{H}_{T}$. We rely on a data-driven estimation of the background using the method of $S_{\mathrm{T}}$ scaling and find the data to be in very good agreement with the estimate. Finally, we use this to compute the first limit on squark mass in the stealth SUSY framework of $1430 \mathrm{GeV}$ at the $95 \%$ confidence level.

\section{References}

[1] M. Lisanti, P. Schuster, M. Strassler and N. Toro, "Study of LHC Searches for a Lepton and Many Jets,” JHEP 1211, 081 (2012) [arXiv:1107.5055 [hep-ph]]. 


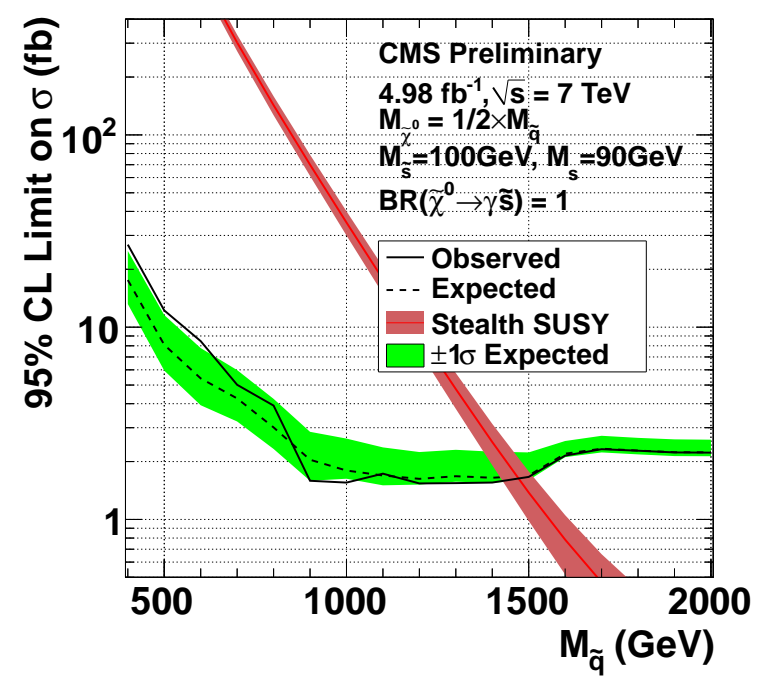

Figure 7: Stealth SUSY cross-section limit at the 95\% CL as a function of squark mass. We show the observed limit, median expected limit, and a band corresponding to \pm 1 SD on the median expected limits. We also show the predicted NLO + NLL cross section from stealth SUSY with a band denoting \pm 1 SD from theoretical uncertainty.

[2] J. Fan, M. Reece and J. T. Ruderman, “Stealth Supersymmetry,” JHEP 1111, 012 (2011) [arXiv:1105.5135 [hep-ph]].

[3] CMS Collaboration, "Interpretation of Searches for Supersymmetry with Simplified Models", CMS Physics Analysis Summary CMS-SUS-11-016, 2012.

[4] M. Kramer, A. Kulesza, R. van der Leeuw, M. Mangano, S. Padhi, T. Plehn and X. Portell, "Supersymmetry production cross sections in $p p$ collisions at $\sqrt{s}=7 \mathrm{TeV}$," arXiv:1206.2892 [hep-ph].

[5] S. Chatrchyan et al. [CMS Collaboration], "Search for microscopic black holes in $p p$ collisions at $\sqrt{s}=7$ TeV,” JHEP 1204, 061 (2012) [arXiv:1202.6396 [hep-ex]]. 